\title{
An Algorithm for Reconstructing Special Lattice Sets from Their Approximate X-Rays ${ }^{\star}$
}

\author{
Sara Brunetti ${ }^{1}$, Alain Daurat ${ }^{2}$, and Alberto Del Lungo ${ }^{3}$ \\ 1 Dipartimento di Sistemi e Informatica \\ Via Lombroso 6/17, Firenze, 50134, Italy \\ brunetti@dsi.unifi.it \\ ${ }^{2}$ Laboratoire de Logique et d'Informatique de Clermont-1 (LLAIC1) \\ Ensemble Universitaire des Cézeaux \\ B.P. $n^{\circ}$ 86, 63172 Aubière Cedex, France \\ daurat@llaic.u-clermont1.fr \\ 3 Dipartimento di Matematica, Via del Capitano 15, 53100, Siena, Italy \\ dellungo@unisi.it
}

\begin{abstract}
We study the problem of reconstructing finite subsets of the integer lattice $\mathbb{Z}^{2}$ from their approximate X-rays in a finite number of prescribed lattice directions. We provide a polynomial-time algorithm for reconstructing Q-convex sets from their "approximate" X-rays. A Qconvex set is a special subset of $\mathbb{Z}^{2}$ having some convexity properties. This algorithm can be used for reconstructing convex subsets of $\mathbb{Z}^{2}$ from their exact X-rays in some sets of four prescribed lattice directions, or in any set of seven prescribed mutually nonparallel lattice directions.
\end{abstract}

\section{Introduction}

The problem of reconstructing two-dimensional lattice sets from their X-rays has been studied in discrete mathematics and applied in several areas. In this context, a two-dimensional lattice set is a finite subsets of the integer lattice $\mathbb{Z}^{2}$, and an $X$-ray of a set in a direction $u$ is a function giving the number of its points on each line parallel to $u$. This problem is the basic reconstruction problem in discrete tomography [10] and it has various interesting applications in image processing [15], statistical data security [12], biplane angiography [14] and reconstructing crystalline structures from X-rays taken by an electron microscope [13]. The computational complexity of this reconstruction problem is studied in [9]. It is shown that the question of reconstructing two-dimensional lattice sets from their X-rays in a set of $m \geq 3$ pairwise nonparallel directions is $\mathbb{N} \mathbb{P}$-hard. In most practical applications we have some a priori properties about the sets to be reconstructed. The algorithms can take advantage of this information to reconstruct the set. Mathematically, these properties can be described

\footnotetext{
* This work is partially supported by MURST project: Modelli di calcolo innovativi: metodi sintattici e combinatori. and by the University Siena project: Problemi Inversi Discreti: Tomografia Discreta
} 
in terms of a subclass of subsets of $\mathbb{Z}^{2}$ to which the solution must belong. For instance, there are polynomial-time algorithms to reconstruct $h v$-convex polyominoes (i.e., two-dimensional lattice subsets which are 4-connected and convex in the horizontal and vertical directions) from their X-rays in horizontal and vertical directions [2,5]. The class of convex lattice subsets (i.e., finite subsets $F$ with $F=\mathbb{Z}^{2} \cap \operatorname{conv} F$ ) is another well-known and studied class in discrete tomography. Gardner and Gritzmann [8] proved that the X-rays in four suitable or any seven prescribed mutually nonparallel lattice directions uniquely determine all the convex lattice subsets. The complexity of the reconstruction problem on this class is an open problem raised by Gritzmann during the workshop: Discrete Tomography: Algorithms and Complexity (1997).

In this paper, we study the problem of reconstructing Q-convex sets from their X-rays in a finite number of prescribed directions. The Q-convexity is a weak convexity property linked to a finite number of directions. The class of Qconvex sets contains all the convex lattice subsets. We provide a polynomial-time algorithm for reconstructing Q-convex sets from their "approximate" X-rays. This means that, the algorithm decides whether or not there is a Q-convex set whose X-rays all lie within prescribed bounds. If there is at least one Q-convex set having X-rays lying within these bounds, the algorithm reconstructs one of them in polynomial time. Boufkhad et al. [3] studied the problem of reconstructing $h, v$-convex polyominoes from their "approximate" horizontal and vertical Xrays. We show that our algorithm solves this problem in polynomial time. The algorithm can be used for reconstructing Q-convex sets from their "exact" Xrays. A greedy algorithm for solving this problem has been defined in [4], and our new approach is faster than this algorithm for a number of directions equal to two and three. We point out that recently, it is proved in [6] that the uniqueness Gardner's and Gritzmann's results can be extended to the class of Q-convex sets. From this uniqueness result for Q-convex sets it follows that our algorithm can be used for reconstructing convex lattice subsets from their exact X-rays in some sets of four prescribed lattice directions, or in any set of seven prescribed mutually nonparallel lattice directions. This means that, our algorithm and the one defined in [4] solve Gritzmann's problem for these special sets of directions.

\section{A Reconstruction Algorithm for Two X-Rays}

In this section, we are going to define an algorithm for reconstructing Q-convex sets from their approximate X-rays in two directions. The basic idea of the algorithm is to determine a polynomial transformation of our problem to the 2-Satisfiability problem which can be solved in linear time $[1,2,5]$.

\subsection{Definitions and Preliminaries}

Let $p$ and $q$ be two independent linear forms on $\mathbb{Q}^{2}$. We can assume that: $p(x, y)=a x+b y$ and $q(x, y)=c x+d y$ with $a, b, c, d \in \mathbb{Z}, a d-b c \neq 0$, $\operatorname{gcd}(a, b)=1, \operatorname{gcd}(c, d)=1$. Assuming that $M=\left(x_{M}, y_{M}\right)$, we denote $p\left(x_{M}, y_{M}\right)$ 
by $p(M)$. The $X$-ray of a lattice set $F$ along direction $p(M)=$ const is the function $X_{p} F: \mathbb{Z} \rightarrow \mathbb{N}_{0}$ defined by:

$$
X_{p} F(i)=\operatorname{card}(\{N \in F \mid p(N)=i\}) .
$$

This, in turn, means that an X-ray of a lattice set $F$ in a direction $p$ is a function giving the number of points of $F$ on each line parallel to this direction (see Fig. 2(a)). We define four zones around a point $M$ of $\mathbb{Z}^{2}$ (see Fig. 1) as follows:

$$
\begin{aligned}
& Z_{0}(M)=\left\{N \in \mathbb{Z}^{2} \mid p(N) \leq p(M) \text { and } q(N) \leq q(M)\right\} \\
& Z_{1}(M)=\left\{N \in \mathbb{Z}^{2} \mid p(N) \geq p(M) \text { and } q(N) \leq q(M)\right\} \\
& Z_{2}(M)=\left\{N \in \mathbb{Z}^{2} \mid p(N) \geq p(M) \text { and } q(N) \geq q(M)\right\} \\
& Z_{3}(M)=\left\{N \in \mathbb{Z}^{2} \mid p(N) \leq p(M) \text { and } q(N) \geq q(M)\right\}
\end{aligned}
$$

We can now introduce the definition of Q-convex set around two directions.

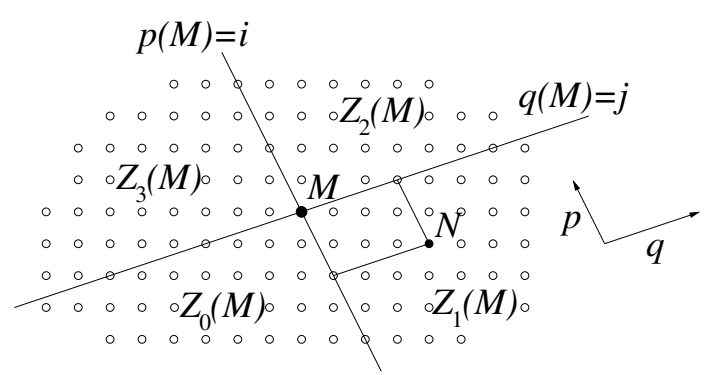

Fig. 1. The four zones around a point $M$ of $\mathbb{Z}^{2}$

Definition 1. A lattice set $F$ is Q-convex around $p$ and $q$ if and only if for each $M \notin F$ there exists $k$ such that $Z_{k}(M) \cap F=\emptyset$ for $k \in\{0,1,2,3\}$.

By the definition, if there is at least one point of $F$ in every zone $Z_{0}(M), Z_{1}(M)$, $Z_{2}(M)$ and $Z_{3}(M)$, the point $M$ has to belong to $F$. Fig. 2(a) shows some examples of Q-convex sets. We point out that, from the definition it follows that a Q-convex set around $p$ and $q$ is a discrete set which is convex along $p$ and $q$. A discrete set $F$ is convex along $p$ if for each pair of points $(M, N)$ of $F$ such that $p(M)=p(N)$, the discrete segment $[M N] \cap \mathbb{Z}^{2}$ is contained in $F$. Let us now take the following problem into consideration.

\section{Problem 1. Approximate Consistency with two directions}

Instance: four vectors $P=\left(p_{1}, \ldots, p_{n}\right), P^{\prime}=\left(p_{1}^{\prime}, \ldots, p_{n}^{\prime}\right), Q=\left(q_{1}, \ldots, q_{m}\right)$, $Q^{\prime}=\left(q_{1}^{\prime}, \ldots, q_{m}^{\prime}\right)$ whose elements are no-negative integer numbers and $p_{1}, p_{n}$, $p_{1}^{\prime}, p_{n}^{\prime}, q_{1}, q_{m}, q_{1}^{\prime}, q_{m}^{\prime}$ are positive integer numbers.

Question: is there a Q-convex set $F$ around $p$ and $q$ such that $p_{i} \leq X_{p} F(i) \leq p_{i}^{\prime}$ for $i=1, \ldots, n$ and $q_{j} \leq X_{q} F(j) \leq q_{j}^{\prime}$ for $j=1, \ldots, m$ ? 

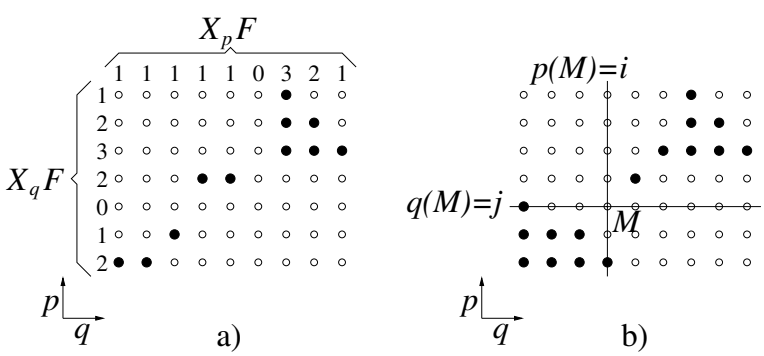

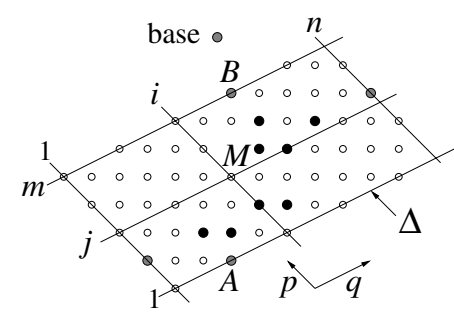

c)

Fig. 2. a) A Q-convex set around $p(x, y)=x$ and $q(x, y)=y$. b) A set which is not Qconvex set around $p(x, y)=x$ and $q(x, y)=y$. c) A Q-convex set around $p(x, y)=x+y$ and $q(x, y)=x-2 y$

The problem is to decide whether or not there is a Q-convex set around $p$ and $q$ whose X-rays in these two directions lie within prescribed bounds. If $P=P^{\prime}$ and $Q=Q^{\prime}$, we have the Exact Consistency problem with two directions.

In the following subsections, we determine a polynomial transformation of Problem 1 to the 2-Satisfiability problem (2SAT).

\subsection{Q-Convexity}

The intersection of the $p$-line $p(M)=i$ with the $q$-line $q(M)=j$ is not always in $\mathbb{Z}^{2}$. It is easy to prove that the point $M$ intersection of $p(M)=i$ with $q(M)=j$ belongs to $\mathbb{Z}^{2}$ if and only if $j \equiv \kappa i(\bmod \delta)$, where: $\delta=|a d-b c|$, $\kappa=(c u+d v) \operatorname{sign}(a d-b c)(\bmod \delta)$ and $a u+b v=1$ (see Fig. 3(a) and [7]).

Without any loss of generality, we can assume that a Q-convex set $F$ around $p$ and $q$ whose X-rays are such that: $p_{i} \leq X_{p} F(i) \leq p_{i}^{\prime}$ and $q_{j} \leq X_{q} F(j) \leq q_{j}^{\prime}$ for all $i, j$, is contained in the lattice parallelogram:

$$
\Delta=\left\{N \in \mathbb{Z}^{2} \mid 1 \leq p(N) \leq n \text { and } 1 \leq q(N) \leq m\right\}
$$

We denote the point $M \in \Delta$ intersection of $p(M)=i$ with $q(M)=j$ by $M=(i, j)$ (see Fig. $2(\mathrm{c}))$. Let $K=\{0,1,2,3\}$. We associate four boolean variables $V_{k}(M)$, with $k \in K$, at every point $M \in \Delta$ (i.e., one variable for each zone $\left.Z_{k}(M)\right)$. The idea of the algorithm is to build a 2SAT formula APPROX on the variables $\left(V_{k}(M)\right)_{k \in K, M \in \Delta}$ so that there is a solution $F$ of Problem 1 if and only if APPROX is satisfiable. If there is an evaluation $V$ of the variables $\left(V_{k}(M)\right)_{k \in K, M \in \Delta}$ satisfying APPROX, the corresponding lattice set $F$ solving Problem 1 is defined by function $\Phi$ as follows:

$$
F=\Phi(V) \text { iff } F=\left\{M \in \Delta \mid \neg V_{k}(M) \text { is true, } \forall k \in K\right\},
$$

where $\neg V_{k}(M)$ is true if and only if $V_{k}(M)$ is false. Conversely, if $F$ is a subset of $\Delta$ solving Problem 1, the corresponding evaluation $V$ of the variables $\left(V_{k}(M)\right)_{k \in K, M \in \Delta}$ satisfying APPROX is defined by function $\Psi$ as follows:

$$
V=\Psi(F) \text { iff } V_{k}(M)=" Z_{k}(M) \cap F=\varnothing ", \text { with } k \in K, M \in \Delta .
$$


We assume that all literals outside $\Delta$ are true. The boolean formula APPROX is made up of three sets of clauses expressing: the Q-convexity (QCONV), a lower bound (LB) and an upper bound (UB) on the X-rays. The Q-convexity can be expressed with the boolean variables by the formulas:

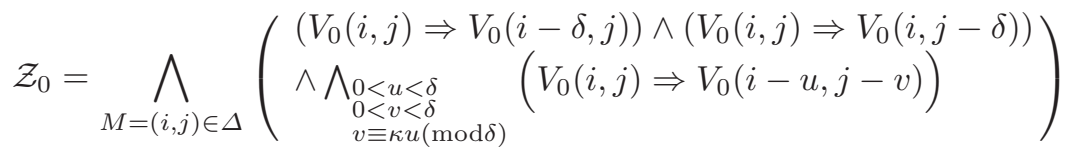

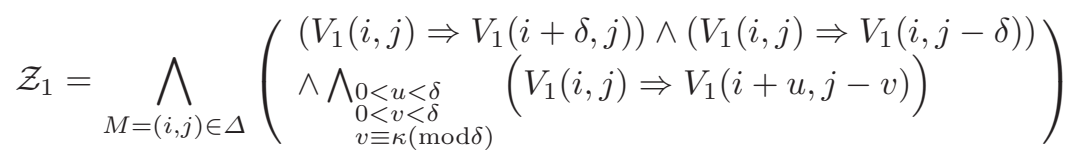

$$
\begin{aligned}
& \mathcal{Z}_{2}=\bigwedge_{M=(i, j) \in \Delta}\left(\begin{array}{l}
\left(V_{2}(i, j) \Rightarrow V_{2}(i+\delta, j)\right) \wedge\left(V_{2}(i, j) \Rightarrow V_{2}(i, j+\delta)\right) \\
\wedge \bigwedge_{\substack{0<u<\delta \\
0<v<\delta \\
v \equiv \kappa u(\bmod \delta)}}\left(V_{2}(i, j) \Rightarrow V_{2}(i+u, j+v)\right)
\end{array}\right)
\end{aligned}
$$

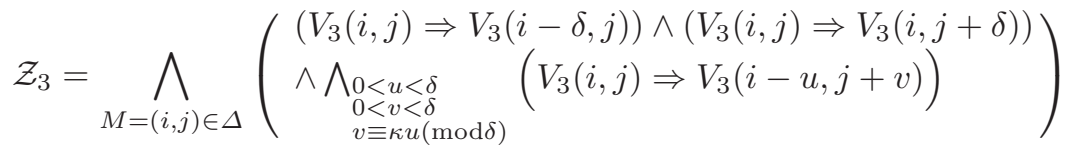

The points in the grey zone around $M=(i, j)$ in Fig. 3(a) are the points of $\Delta$ used in $\mathcal{Z}_{0}, \mathcal{Z}_{1}, \mathcal{Z}_{2}$ and $\mathcal{Z}_{3}$ (i.e., the points $(i \pm \delta, j \pm \delta),(i \pm u, j \pm v)$ with $0<u<\delta, 0<v<\delta$ ). Let us denote $\mathcal{Z}_{0} \wedge \mathcal{Z}_{1} \wedge \mathcal{Z}_{2} \wedge \mathcal{Z}_{3}$ by QCONV.

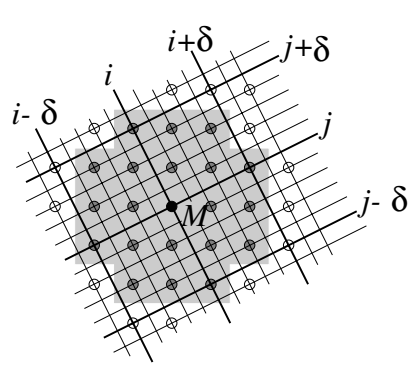

a)

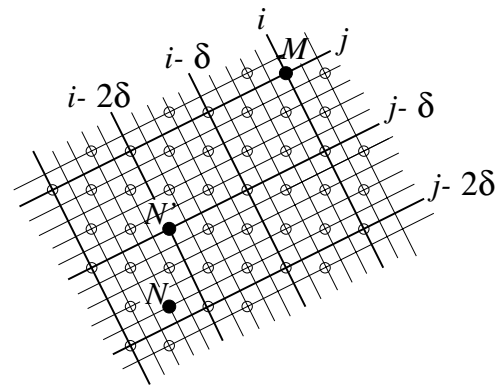

b)

Fig. 3. The points around $M$ used in $\mathcal{Z}_{0}, \mathcal{Z}_{1}, \mathcal{Z}_{2}$ and $\mathcal{Z}_{3}$ with $p(x, y)=2 x+y$ and $q(x, y)=x-2 y$

Lemma 1. Let $V$ be an evaluation of the variables $\left(V_{k}(M)\right)_{k \in K, M \in \Delta}$ satisfying $Q C O N V$. If $M \in \Delta$ and $V_{k}(M)$ is true, then $V_{k}(N)$ is true for all $N \in Z_{k}(M)$.

Proof. Assume that $k=0$ and let $M=\left(i_{M}, j_{M}\right)$. We have: $V_{0}(i, j) \Rightarrow V_{0}(i-\delta, j)$ and $V_{0}(i, j) \Rightarrow V_{0}(i, j-\delta)$ are satisfied, for all $i, j$. Therefore, by induction we can prove that $V_{0}\left(i_{M}-k \delta, j_{M}-l \delta\right)$ is true, for all $k, l \in \mathbb{N}$. Let $N$ be a point 
of $Z_{0}(M)$. Let $N^{\prime}$ be the point of $\Delta$ such that:

$$
\frac{p(M)-p\left(N^{\prime}\right)}{\delta}=\left\lfloor\frac{p(M)-p(N)}{\delta}\right\rfloor, \quad \frac{q(M)-q\left(N^{\prime}\right)}{\delta}=\left\lfloor\frac{q(M)-q(N)}{\delta}\right\rfloor
$$

By the previous statement we have $V_{0}\left(N^{\prime}\right)$ is true (see Fig. 3(b)) and, since the formula $V_{0}\left(N^{\prime}\right) \Rightarrow V_{0}(N)$ is in $\mathcal{Z}_{0}$, we finally obtain $V_{0}(N)$ is true. We proceed in the same way for $k$ equal to 1,2 and 3 .

Thus, we can characterize the Q-convexity by means of the formula QCONV.

\section{Lemma 2.}

- For any set $F \subset \Delta$ the evaluation $V=\Psi(F)$ of the boolean variables $\left(V_{k}(M)\right)_{k \in K, M \in \Delta}$ satisfies the formula $Q C O N V$.

- If an evaluation $V$ of the boolean variables $\left(V_{k}(M)\right)_{k \in K, M \in \Delta}$ satisfies the formula $Q C O N V$, then $F=\Phi(V)$ is $Q$-convex around $p$ and $q$.

Proof.

- Assume that $V=\Psi(F)$ does not satisfy QCONV. By this assumption, there exists $k$ such that at least a clause of $\mathcal{Z}_{k}$ is not satisfied. Then $V_{k}(M)$ and $\neg V_{k}(N)$ are true, where $N \in Z_{k}(M)$. As a consequence, $Z_{k}(M) \cap F=$ $\varnothing, Z_{k}(N) \cap F \neq \varnothing$ and $Z_{k}(N) \subset Z_{k}(M)$. We got a contradiction and so $V=\Psi(F)$ satisfies QCONV.

- The second statement is just a consequence of Lemma 1. If $M \notin F$, by the definition of $\Phi$ there exists $k$ such that $V_{k}(M)$ is true. Therefore, by Lemma 1 , we have $V_{k}(N)$ for each $N \in Z_{k}(M)$. Consequently, $Z_{k}(M) \cap F=\varnothing$.

\subsection{A Lower Bound}

Now we want to express that X-ray values of a lattice set in the direction $p$ are greater than some prescribed integers. Let us take the line $p(M)=i$ into consideration. Let $\operatorname{qmin}_{i}=\min \{j \mid(i, j) \in \Delta\}$ and $\operatorname{qmax}_{i}=\max \{j \mid(i, j) \in \Delta\}$. Notice that, if $\delta \neq 1$ these numbers are not always equal to 1 and $m$. We define the formula $\operatorname{LB}(p, i, l)$ in the following way:

$$
\begin{array}{ll}
\operatorname{LB}(p, i, l)=T R U E & \text { if } l=0 \\
\operatorname{LB}(p, i, l)=\left(\begin{array}{cc}
\bigwedge_{1 \leq j \leq m-\delta l} \underset{j \equiv \kappa i}{(\bmod \delta)}\left(L_{1}(j) \wedge L_{2}(j) \wedge L_{3}(j) \wedge L_{4}(j)\right) \\
\wedge L_{1}^{\prime} \wedge L_{2}^{\prime} \wedge L_{3}^{\prime} \wedge L_{4}^{\prime}
\end{array}\right) & \text { otherwise }
\end{array}
$$


where

$$
\begin{aligned}
L_{1}(j) & =V_{0}(i, j) \Rightarrow \neg V_{2}(i, j+\delta l) \\
L_{2}(j) & =V_{0}(i, j) \Rightarrow \neg V_{3}(i, j+\delta l) \\
L_{3}(j) & =V_{1}(i, j) \Rightarrow \neg V_{2}(i, j+\delta l) \\
L_{4}(j) & =V_{1}(i, j) \Rightarrow \neg V_{3}(i, j+\delta l) \\
L_{1}^{\prime} & =\neg V_{2}\left(i, \operatorname{qmin}_{i}+\delta(l-1)\right) \\
L_{2}^{\prime} & =\neg V_{3}\left(i, \operatorname{qmin}_{i}+\delta(l-1)\right) \\
L_{3}^{\prime} & =\neg V_{0}\left(i, \operatorname{qmax}_{i}-\delta(l-1)\right) \\
L_{4}^{\prime} & =\neg V_{1}\left(i, \operatorname{qmax}_{i}-\delta(l-1)\right)
\end{aligned}
$$

\section{Lemma 3.}

- If a lattice set $F$ is Q-convex around $p$ and $q$ and its $X$-ray along $p$ is such that $X_{p} F(i) \geq l$, then the evaluation $V=\Psi(F)$ of the variables $\left(V_{k}(M)\right)_{k \in K, M \in \Delta}$ satisfies $Q C O N V \wedge \operatorname{LB}(p, i, l)$.

- If an evaluation $V$ of the variables $\left(V_{k}(M)\right)_{k \in K, M \in \Delta}$ satisfies $Q C O N V \wedge$ $\mathrm{LB}(p, i, l)$, then the $X$-ray of $F=\Phi(V)$ along $p$ is such that $X_{p} F(i) \geq l$.

We do not show the proof of this Lemma for brevity's sake. We define the formula $\operatorname{LB}(q, j, l)$ for the lines in the direction $q$ in a similar way.

\subsection{An Upper Bound}

Now we want to express that X-ray values of a lattice set in the direction $q$ are smaller than some prescribed integers. For this upper bound, we need to fix two points $A$ and $B$ such that $p(A)=1$ and $p(B)=n$. We call bases these two points (see Fig. 2(c)). Let us take the line $q(M)=j$ into consideration. We introduce the formula:

$$
\mathrm{UB}(q, j, l, A, B)=\mathrm{IN}(A) \wedge \mathrm{IN}(B) \wedge \bigwedge_{\substack{1 \leq i \leq n-\delta l \\ j \equiv \kappa i}} U(i)
$$

where : $\quad \operatorname{IN}(M)=\neg V_{0}(M) \wedge \neg V_{1}(M) \wedge \neg V_{2}(M) \wedge \neg V_{3}(M)$ and
a) If $j \leq \min \{q(A), q(B)\}$,
$U(i)=\neg V_{0}(i, j) \Rightarrow V_{1}(i+\delta l, j)$
b) If $q(A) \leq j \leq q(B)$
$U(i)=\neg V_{3}(i, j) \Rightarrow V_{1}(i+\delta l, j)$
c) If $q(B) \leq j \leq q(A)$
$U(i)=\neg V_{0}(i, j) \Rightarrow V_{2}(i+\delta l, j)$
b) If $j \geq \max \{q(A), q(B)\}$
$U(i)=\neg V_{3}(i, j) \Rightarrow V_{2}(i+\delta l, j)$ 


\section{Lemma 4.}

- If a lattice set $F$ containing the bases $A, B$ is Q-convex around $p$ and $q$, and its $X$-ray along $q$ is such that $X_{q} F(j) \leq l$, then the evaluation $V=\Psi(F)$ of the variables $\left(V_{k}(M)\right)_{k \in K, M \in \Delta}$ satisfies $Q C O N V \wedge \mathrm{UB}(q, j, l, A, B)$.

- If an evaluation $V$ of the boolean variables $\left(V_{k}(M)\right)_{k \in K, M \in \Delta}$ satisfies the formula $Q C O N V \wedge \mathrm{UB}(q, j, l, A, B)$, then $F=\Phi(V)$ contains the bases $A, B$, and its $X$-ray along $q$ is such that $X_{q} F(j) \leq l$.

We do not show the proof of this Lemma for brevity's sake. We define the formula $\mathrm{UB}(p, i, l, A, B)$ for the lines in the direction $p$ in a similar way .

\subsection{The Reconstruction Algorithm}

Let $\left(P, P^{\prime}, Q, Q^{\prime}\right)$ be an instance of Problem 1. We fix four bases $A, B, C, D$ such that $p(A)=1, p(B)=n, q(C)=1, q(D)=m$ and then we build the formula:

$$
\begin{aligned}
& \operatorname{APPROX}\left(P, P^{\prime}, Q, Q^{\prime}, A, B, C, D\right)=Q C O N V \wedge \\
& \bigwedge_{1 \leq i \leq n}\left(\mathrm{LB}\left(p, i, p_{i}\right) \wedge \mathrm{UB}\left(p, i, p_{i}^{\prime}, C, D\right)\right) \wedge \bigwedge_{1 \leq j \leq m}\left(\mathrm{LB}\left(q, j, q_{j}\right) \wedge \mathrm{UB}\left(q, j, q_{j}^{\prime}, A, B\right)\right)
\end{aligned}
$$

As a consequence of Lemmas 2,3 and 4, we get:

Theorem 1. $\operatorname{APPROX}\left(P, P^{\prime}, Q, Q^{\prime}, A, B, C, D\right)$ is satisfiable if and only if there is a $Q$-convex set $F$ around $p$ and $q$ containing the bases $A, B, C, D$ and having $X$-rays along $p$ and $q$ such that $p_{i} \leq X_{p} F(i) \leq p_{i}^{\prime}$, for $i=1, \ldots, n$, and $q_{j} \leq$ $X_{q} F(j) \leq q_{j}^{\prime}$, for $j=1, \ldots, m$.

Since $\operatorname{APPROX}\left(P, P^{\prime}, Q, Q^{\prime}, A, B, C, D\right)$ is a boolean formula in conjunctive normal form with at most two literals in each clause, from Theorem 1 we have a transformation of Problem 1 to 2SAT problem. The algorithm chooses four bases $A, B, C, D$, and builds $\operatorname{APPROX}\left(P, P^{\prime}, Q, Q^{\prime}, A, B, C, D\right)$. Each formula $\operatorname{APPROX}\left(P, P^{\prime}, Q, Q^{\prime}, A, B, C, D\right)$ has size $O(m n)$ and can be constructed in $O(m n)$ time. This is a 2SAT formula and so it can be solved in $\mathrm{O}(m n)$ time (see [1]). If the formula is satisfiable and $V$ is the evaluation of the boolean variables, then $F=\Phi(V)$ is solution of Problem 1. On the contrary, the reconstruction attempt fails and the algorithm chooses a different position of the four bases $A, B, C, D$, and repeats the procedure. The number of reconstruction attempts is bounded by the number of different positions of the four bases $A, B, C, D$, and this is at most $m^{2} n^{2}$. Consequently:

Corollary 1. Problem 1 can be solved in $O\left(m^{3} n^{3}\right)$ time.

Remark 1. An 8-connected $h v$-convex set is a Q-convex set around $p(x, y)=x$ and $q(x, y)=y$ with at least one point in each row and column. This class of lattice sets is a well-know generalization of the class of $h v$-convex polyominoes $[2,5]$ which are 4-connected and convex in horizontal and vertical directions. Boufkhad et al. [3] studied the "approximate consistency with two directions" 
problem on the class of $h v$-convex polyominoes. In detail, given a pair of vectors $V=\left(v_{1}, \ldots, v_{n}\right)$ and $H=\left(h_{1}, \ldots, h_{m}\right)$, they want to reconstruct an $h v$-convex polyomino whose X-rays along vertical and horizontal directions are such that: $\left|X_{p} F(i)-v_{i}\right| \leq 1$ for $i=1, \ldots, n$ and $\left|X_{q} F(j)-h_{j}\right| \leq 1$ for $j=1, \ldots, m$ (i.e., $P=V-1, P^{\prime}=V+1, Q=H-1$ and, $\left.Q^{\prime}=H+1\right)$. Our algorithm solves this problem in polynomial time on the classes of 8-connected $h v$-convex sets and $h v$-convex polyominoes (with an extra condition on the boolean variables). We point out that the goal of Boufkhad et al. is to solve the corresponding optimization problem. They want to reconstruct $h v$-convex polyominoes from these "approximate" horizontal and vertical X-rays and such that the sum of the absolute differences is minimum. By means of a SAT solver, the authors defined a heuristic algorithm for solving this problem. We do not know at this time if our algorithm can be used for solving this optimization problem in polynomial time.

Remark 2. Mirsky [11] proved that "approximate consistency with two directions" problem on the class of all lattice sets can be solved in polynomial time.

The Exact Reconstruction. If $P=P^{\prime}$ and $Q=Q^{\prime}$, Problem 1 becomes the Exact Consistency problem with two directions on the Q-convex sets around $p$ and $q$. Notice that, $\sum_{i=1}^{n} p_{i}=\sum_{j=1}^{n} q_{j}$ is a necessary condition. This problem has been studied in [4] and the authors propose a greedy algorithm whose computational cost is $O\left(m^{2} n^{2}(m+n) \min \left\{m^{2}, n^{2}\right\}\right)$. We are going to show an algorithm which is faster than the algorithm defined in [4]. Let $A, B$ be two bases such that $p(A)=1$ and $p(B)=n$. We build the following formula:

$$
\operatorname{EXACT}(P, Q, A, B)=Q C O N V \bigwedge_{1 \leq i \leq n} \operatorname{LB}\left(p, i, p_{i}\right) \bigwedge_{1 \leq j \leq m} \mathrm{UB}\left(q, j, q_{j}, A, B\right) .
$$

Proposition 1. $\operatorname{EXACT}(P, Q, A, B)$ is satisfiable if and only if there is a $Q$ convex set $F$ around $p$ and $q$ containing $A, B$ and having $X$-rays along $p$ and $q$ such that $X_{p} F(i)=p_{i}$, for $i=1, \ldots, n$, and $X_{q} F(j)=q_{j}$, for $j=1, \ldots, m$.

Proof.

- Assume that $\operatorname{EXACT}(P, Q, A, B)$ is satisfied by an evaluation $V$ of

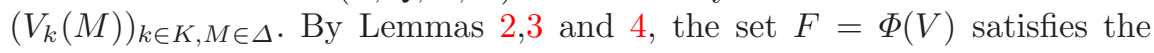
conditions: $A, B \in F, X_{p} F(i) \geq p_{i}$ and $X_{q} F(j) \leq q_{j}$ for all $i, j$. Then,

$$
\sum_{j=1}^{m} q_{j} \geq \sum_{j=1}^{m} X_{q} F(j)=\sum_{i=1}^{n} X_{p} F(i) \geq \sum_{i=1}^{m} p_{i}
$$

and, since $\sum_{j=1}^{m} q_{j}=\sum_{i=1}^{n} p_{i}, X_{p} F(i)=p_{i}$ and $X_{q} F(j)=q_{j}$ for all $i, j$.

- If $F$ is Q-convex around $p$ and $q$ and satisfies $A, B \in F, X_{p} F(i)=p_{i}$, and $X_{q} F(j)=q_{j}$, by Lemmas 2,3 and 4 , the evaluation $V=\Psi(F)$ satisfies $\operatorname{EXACT}(P, Q, A, B)$. 
The number of reconstruction attempts for the exact consistency problem is bounded by the number of different positions of the two bases $A, B$, and this is at $\operatorname{most} \min \left\{m^{2}, n^{2}\right\}$. Consequently:

Corollary 2. Exact Consistency problem is solved in $O\left(m n \min \left\{m^{2}, n^{2}\right\}\right)$ time.

\section{More than Two Directions}

We now outline an algorithm for reconstructing Q-convex sets from their X-rays in more than two directions. Let us introduce a definition of Q-convex set around more than two directions. Let $U$ be a set of $d$ directions $\left\{\boldsymbol{u}_{h}=\left(a_{h}, b_{h}\right)_{h=1}^{d}\right\}$ (i.e., pairs of coprime integers, with $\left.b_{h} \geq 0\right)$. The linear form corresponding to vector $\boldsymbol{u}_{h}=\left(a_{h}, b_{h}\right)$ is $u_{h}(x, y)=b_{h} x-a_{h} y$. Given two directions $u_{i}, u_{j} \in U$, we define four zones $Z_{k}^{(i, j)}(M)$ around every $M \in \mathbb{Z}^{2}$ as in the previous section. Therefore, there are $2 d(d-1)$ zones for each $M \in \mathbb{Z}^{2}$ and we are going to select $2 d$ of these zones. A point $M$ of a line in direction $u_{h}$ splits it into the following two semi-lines having origin in $M$ :

$$
\begin{aligned}
& s_{h}^{+}(M)=\left\{N \in \mathbb{Z}^{2} \mid u_{h}(N)=u_{h}(M) \text { and } \boldsymbol{u}_{h} \cdot \overrightarrow{O N} \geq \boldsymbol{u}_{h} \cdot \overrightarrow{O M}\right\} \\
& s_{h}^{-}(M)=\left\{N \in \mathbb{Z}^{2} \mid u_{h}(N)=u_{h}(M) \text { and } \boldsymbol{u}_{h} \cdot \overrightarrow{O N} \leq \boldsymbol{u}_{h} \cdot \overrightarrow{O M}\right\}
\end{aligned}
$$

where "." denotes the scalar product of two vectors and $O$ is any origin point.

Definition 2. An almost-semi-plane (or $A S P$ ) along $U$ is a zone $Z_{k}^{(i, j)}(M)$ such that for each direction $u_{h}$ of $U$ only one of the two semi-lines $s_{h}^{+}(M), s_{h}^{-}(M)$ is contained in $Z_{k}^{(i, j)}(M)$.

Let $\Pi_{0}(M)$ be the ASP containing $s_{h}^{+}(M)$ for each $h=1, \ldots, d$. We denote the other almost-semi-planes encountered clockwise around $M$ from $\Pi_{0}(M)$ by $\Pi_{1}(M), \ldots, \Pi_{2 d-1}(M)$. For example, let $U=\left\{u_{1}, u_{2}, u_{3}\right\}$, with $u_{1}=y, u_{2}=$ $x, u_{3}=x+y$. The six ASP around a point $M$ are: $\Pi_{0}(M)=Z_{2}^{(1,3)}(M), \Pi_{1}(M)=$ $Z_{2}^{(2,3)}(M), \Pi_{2}(M)=Z_{1}^{(1,2)}(M), \Pi_{3}(M)=Z_{0}^{(1,3)}(M), \Pi_{4}(M)=Z_{0}^{(2,3)}(M)$ and $\Pi_{5}(M)=Z_{3}^{(1,2)}(M)$ (see Fig. 4). Now we can generalize the Q-convexity to any set of directions:

Definition 3. A lattice set $F$ is strongly Q-convex around $U$ if and only if for each $M \notin F$ there exists an $A S P \Pi_{k}(M)$ around $M$ such that $F \cap \Pi_{k}(M)=\varnothing$.

Let us consider the approximate consistency problem on this class.

Problem 2. Approximate Consistency with more than two directions Instance: $2 d$ vectors $P_{1}=\left(p_{1,1}, \ldots, p_{1, n_{1}}\right), P_{1}^{\prime}=\left(p_{1,1}^{\prime}, \ldots, p_{1, n_{1}}^{\prime}\right), \ldots, P_{d}=$ $\left(p_{d, 1}, \ldots, p_{d, n_{d}}\right)$ and $P_{d}^{\prime}=\left(p_{d, 1}^{\prime}, \ldots, p_{d, n_{d}}^{\prime}\right)$ whose elements are no-negative integer numbers and $p_{1,1}, p_{1, n_{1}}, p_{1,1}^{\prime}, p_{1, n_{1}}^{\prime}, \ldots, p_{d, 1}, p_{d, n_{d}}, p_{d, 1}^{\prime}, p_{d, n_{d}}^{\prime}$ are positive integer numbers.

Question: is there a strongly Q-convex set $F$ around $U$ such that: $p_{h, i} \leq X_{u_{h}} F(i) \leq p_{h, i}^{\prime}$ for $i=1, \ldots, n_{h}$ and $h=1, \ldots, d$ ? 


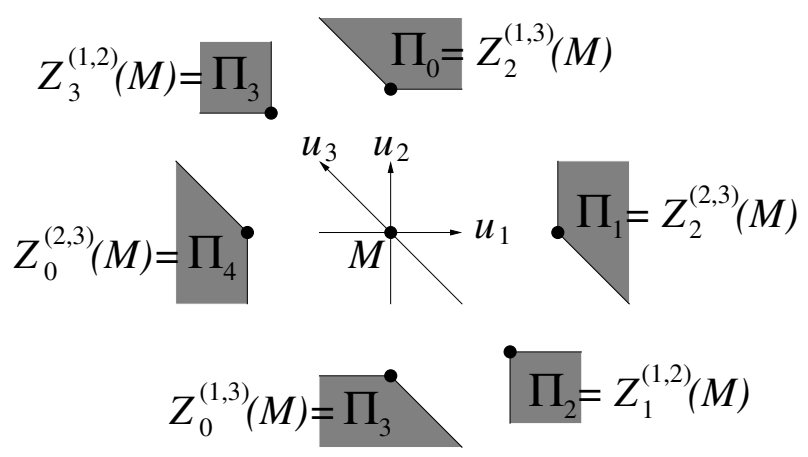

Fig. 4. The six ASP around $M$, with $U=\left\{u_{1}, u_{2}, u_{3}\right\}, u_{1}=y, u_{2}=x, u_{3}=x+y$

A Q-convex set $F$ around $U$ whose X-rays are such that: $p_{h, i} \leq X_{u_{h}} F(i) \leq p_{h, i}^{\prime}$ for all $h, i$, is contained in the lattice polygon:

$$
\Delta=\left\{N \in \mathbb{Z}^{2} \mid 1 \leq u_{h}(N) \leq n_{h} \forall 1 \leq h \leq d\right\} .
$$

We use the strategy of the previous section, by replacing the zone $Z_{k}(M)$ with $\Pi_{k}(M)$. Assuming that $K=\{0,1, \ldots, 2 d-1\}$, we associate $2 d$ boolean variables $V_{k}(M)$, with $k \in K$, at every point $M$ in $\Delta$ (i.e., one variable for each $\Pi_{k}(M)$ around $\left.M\right)$. We build a 2SAT formula APPROX on the variables

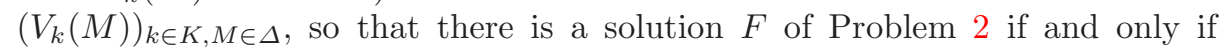

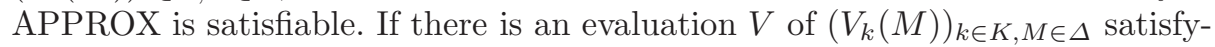
ing APPROX, the corresponding set $F$ solving Problem 2 is $F=\Phi(V)$, with $\Phi$ defined as in the previous section. Conversely, if $F$ is a subset of $\Delta$ solving Problem 1, the corresponding evaluation $V$ of $\left(V_{k}(M)\right)_{k \in K, M \in \Delta}$ satisfying APPROX is $V=\Psi(F)$, with $\Psi$ defined as in the previous section. Since every $\operatorname{ASP} \Pi_{k}(M)$ is equal to a zone $Z_{h}^{(i, j)}(M)$ defined by directions $u_{i}, u_{j}$, it is easy to generalize the formulas to contexts having $n$ directions. The generalization SQCONV of $\mathrm{QCONV}$ is such that $\mathrm{SQCONV}=\mathcal{Z}_{0} \wedge \ldots \wedge \mathcal{Z}_{2 d-1}$, where $\mathcal{Z}_{k}$ corresponds to $\Pi_{k}(M)$ (i.e., $Z_{h}^{(i, j)}(M)$ ). Thus, we have the following extensions of Lemma 2 :

\section{Lemma 5.}

- For any set $F \subset \Delta$ the evaluation $V=\Psi(F)$ of the variables

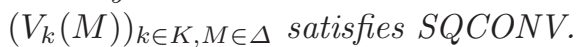

- If an evaluation $V$ of the variables $\left(V_{k}(M)\right)_{k \in K, M \in \Delta}$ satisfies $S Q C O N V$, then $F=\Phi(V)$ is strongly $Q$-convex around $U$.

A lower bound of $l$ on the X-ray of $F$ along the line $u_{h}(M)=i$ is expressed by a 2SAT formula. This formula can be constructed by proceeding as in the twodirections case. We do not describe the construction of the formula $\operatorname{LB}\left(u_{h}, i, l\right)$ for brevity's sake. Finally, we fix $d-1$ pairs of bases for each directions, in order to give an upper bound of $l$ on the X-ray of $F$ in the direction $u_{h}$. The chosen bases 
correspond to the other $d-1$ directions. The upper bound is expressed by the formula UB $\left(u_{h}, i, l, A_{1}, B_{1}, \ldots, A_{h-1}, B_{h-1}, A_{h+1}, B_{h+1}, \ldots, A_{d}, B_{d}\right)$, where $A_{j}, B_{j}$ are the bases of the direction $u_{j}$. This formula is a simple generalization of the upper bound formula for the two-directions case. Let $\left(P_{1}, P_{1}^{\prime}, \ldots, P_{d}, P_{d}^{\prime}\right)$ be an instance of Problem 2. We fix $2 d$ bases $A_{1}, B_{1}, \ldots, A_{d}, B_{d}$ and then we build the following boolean formula:

$\operatorname{APPROX}\left(P_{1}, P_{1}^{\prime}, \ldots, P_{d}, P_{d}^{\prime}, A_{1}, B_{1}, \ldots, A_{d}, B_{d}\right)=S Q C O N V \wedge$
$\bigwedge_{\substack{1 \leq h \leq d \\ 1 \leq i \leq n_{h}}} \mathrm{LB}\left(u_{h}, i, p_{i}\right) \wedge \mathrm{UB}\left(p, i, p_{i}^{\prime}, A_{1}, B_{1}, \ldots, A_{h-1}, B_{h-1}, A_{h+1}, B_{h+1}, \ldots, A_{d}, B_{d}\right)$

We deduce that:

Theorem 2. $\operatorname{APPROX}\left(P_{1}, P_{1}^{\prime} \ldots, A_{d}, B_{d}\right)$ is satisfiable if and only if there is a strongly $Q$-convex set $F$ around $U$ containing the bases $A_{1}, B_{1}, \ldots, A_{d}, B_{d}$ and having $X$-rays along $u_{h}$ such that $p_{h, i} \leq X_{u_{h}} F(i) \leq p_{h, i}^{\prime}$, for $i=1, \ldots, n_{h}$ and $h=1, \ldots, d$.

The algorithm chooses $d$ pair of bases, and builds the 2SAT expression APPROX. Assuming that $n=\max \left\{n_{1}, \ldots, n_{d}\right\}$, we have that each formula APPROX has size $O\left(n^{2}\right)$ and can be constructed in $O\left(n^{2}\right)$ time. The number of reconstruction attempts is bounded by the number of different positions of the $2 d$ bases, and this is at most $n^{2 d}$. Consequently:

Corollary 3. Problem 2 can be solved in $O\left(n^{2 d+2}\right)$ time.

If $P_{h}=P_{h}^{\prime}$ for each $1 \leq h \leq d$, Problem 2 become the exact consistency problem with more than two directions. In this case, we have to choose $d-1$ pair of bases for the upper bound and we complexity of algorithm for solving this problem is $O\left(n^{2 d}\right)$. The convex lattice sets are special Q-convex sets, and so by uniqueness results for Q-convex sets proved in [6], the algorithm can be used for reconstructing convex lattice subsets from their exact X-rays in some sets of four suitable lattice directions, or in any set of seven prescribed mutually nonparallel lattice directions. This means that the two algorithms solve Gritzmann's problem for these special sets of directions.

\section{References}

1. B. Aspvall, M. F. Plass and R. E. Tarjan, A linear-time algorithm for testing the truth of certain quantified Boolean formulas, Information Processing Letters, 83 (1979) 121-123. 114, 120

2. E. Barcucci, A. Del Lungo, M. Nivat and R. Pinzani, Reconstructing convex polyominoes from their horizontal and vertical projections, Theoretical Computer Science, 155 (1996) 321-347. 114, 120

3. Y. Boufkhad, O. Dubois and M. Nivat, Reconstructing (h,v)-convex bidimensional patterns of objects from approximate horizontal and vertical projections, preprint. 114,120 
4. S. Brunetti, A. Daurat, Reconstruction of Discrete Sets From Two or More Projections in Any Direction, Proc. of the Seventh International Workshop on Combinatorial Image Analysis (IWCIA 2000), Caen, (2000) 241-258. 114, 121

5. M. Chrobak, C. Dürr, Reconstructing hv-Convex Polyominoes from Orthogonal Projections, Information Processing Letters, 696 (1999) 283-289. 114, 120

6. A. Daurat, Uniqueness of the reconstruction of Q-convex from their projections, preprint. 114, 124

7. A. Daurat, A. Del Lungo and M. Nivat, The medians of discrete sets according to some linear metrics, Discrete \& Computational Geometry, 23 (2000) 465-483. 116

8. R. J. Gardner and P. Gritzmann, Discrete tomography: determination of finite sets by X-rays, Trans. Amer. Math. Soc., 349 (1997) 2271-2295. 114

9. R. J. Gardner, P. Gritzmann and D. Prangenberg, On the computational complexity of reconstructing lattice sets from their X-rays, Discrete Mathematics, 202 (1999) 45-71. 113

10. A. Kuba and G. T. Herman, Discrete Tomography: A Historical Overview, in Discrete Tomography: Foundations, Algorithms and Applications, editors G. T. Herman and A. Kuba, Birkhauser, Boston, MA, USA, (1999) 3-34. 113

11. L. Mirski, Combinatorial theorems and integral matrices, Journal of Combinatorial Theory, 5 (1968) 30-44. 121

12. R. W. Irving and M. R. Jerrum, Three-dimensional statistical data security problems, SIAM Journal of Computing, 23 (1994) 170-184. 113

13. C. Kisielowski, P. Schwander, F. H. Baumann, M. Seibt, Y. Kim and A. Ourmazd, An approach to quantitative high-resolution transmission electron microscopy of crystalline materials, Ultramicroscopy, 58 (1995) 131-155. 113

14. G. P. M. Prause and D. G. W. Onnasch, Binary reconstruction of the heart chambers from biplane angiographic image sequence, IEEE Transactions Medical Imaging, 15 (1996) 532-559. 113

15. A. R. Shliferstein and Y. T. Chien, Switching components and the ambiguity problem in the reconstruction of pictures from their projections, Pattern Recognition, 10 (1978) 327-340. 113 